\title{
Creencias de los Futuros Maestros sobre la Aptitud Matemática: consideraciones para Promover Procesos de Cambio en la Formación Inicial
}

\author{
Pre-service Teachers' Beliefs about Mathematics Aptitude: considerations \\ to Promote Change Processes in Initial Training
}

\author{
Paula López ${ }^{*}$ \\ Ángel Alsina**
}

\begin{abstract}
Resumen
Los estudios acerca del dominio afectivo en educación matemática señalan que las creencias de los maestros influyen en sus prácticas docentes y, por tanto, en el aprendizaje de los alumnos. Desde este prisma, en este estudio se analizan las creencias sobre la aptitud matemática de 142 estudiantes de primer curso del Grado de Maestro en Educación Infantil y Primaria, que han indicado a través de un cuestionario los factores que piensan que influyen más para ser un buen alumno de matemáticas. Los resultados muestran que los futuros maestros dan mayor importancia a los factores cognitivos $(51,1 \%)$, en segundo lugar a los actitudinales $(27,7 \%) \mathrm{y}$, finalmente, a los procedimentales $(21,1 \%)$. Estos resultados apuntan que en la formación inicial se deberían tratar, como parte del conocimiento didáctico, las creencias sobre la aptitud matemática que dan lugar a una identidad profesional negativa, para poder identificarlas y promover procesos de cambio.
\end{abstract}

Palabras-clave: Aptitud Matemática. Formación Inicial de Maestros de Matemáticas. Identidad Profesional del Maestro de Matemáticas. Sistema de Creencias. Procesos de Cambio.

\begin{abstract}
In studies about the affective domain in Mathematics education, several authors have shown that pre-service teachers' beliefs have a significant influence on their classroom practices and, therefore, on the mathematics learning of their students. From this point, this article analyzes the pre-service teachers' beliefs on mathematical ability from 142 Childhood Education and Primary Education students who have indicated through a questionnaire, the factors that they believe influence the most to be a good student in mathematics. The results show that pre-service teachers give more importance to cognitive factors $(51.1 \%)$, secondly, they consider the attitudinal factors (27.7\%), and at last the procedural factors (21.1\%). These results suggest that, in the context of initial training, as part of didactic knowledge, beliefs that lead to negative professional identity should be analyzed in order to identify and promote change processes.
\end{abstract}

Keywords: Mathematics Aptitude. Mathematics Teacher Training. Mathematical Identity Work. Belief System. Change Processes.

\footnotetext{
* Doctora en Didáctica de las Matemáticas por la Universidad de Barcelona (UB). Profesora asociada de Didáctica de la Matemática en la Universidad de Girona (UdG), Girona, España. Dirección postal: Plaça Sant Domènec, 9, 17071, Girona, España. E-mail: paula.lopez.serentill@ gmail.com.

${ }^{* * *}$ Doctor en Psicología por la Universidad Autónoma de Barcelona (UAB). Profesor agregado de Didáctica de la Matemática en la Universidad de Girona (UdG), Girona, España. Dirección postal: Plaça Sant Domènec, 9, 17071, Girona, España. E-mail: angel.alsina@udg.edu.
} 


\section{Introducción}

Son muchas las situaciones relacionadas con la práctica educativa en las que es útil conocer el sistema de creencias del profesorado sobre la enseñanza y el aprendizaje de las matemáticas, por ejemplo para implicar a los profesores en procesos de cambio (PONTE, 1999; THOMSON, 1992; CARRILLO, 1998; CONTRERAS, 1999; KAASILA et al., 2006, 2008).

Diversos estudios han puesto de manifiesto que las creencias de los maestros ejercen una notable influencia en las prácticas de aula que llevan a cabo, las cuales acaban determinando el aprendizaje de las matemáticas que realizan los alumnos (GROUWS, 1996; CARTER; NOREOOD, 1997; BROWN, 2003; KAYAN, 2007). Una posible interpretación de estos estudios es que los maestros planifican y gestionan la actividad docente en el aula en función de las creencias acerca de las capacidades de sus alumnos, entre otras variables y factores.

Gil y Rico (2003, p. 38), por ejemplo, indican que una de las creencias de los maestros es que

[...] el aprendizaje de las matemáticas se basa en la capacidad intelectual de los alumnos, se expresa por un buen rendimiento y se desarrolla por ejercitación; cuando el profesor aprecia esta situación de aprendizaje, le produce satisfacción (...) el profesor ayuda al alumno que tiene capacidad intelectual en su aprendizaje mediante ejercicios y prácticas.

Estas creencias del profesorado forman parte de la identidad matemática (mathematical identity), que es el resultado de un proceso de interacción y de reflexión individual y social como aprendices y enseñantes de matemáticas, que considera la visión de las matemáticas como conocimiento, creencias, concepciones, actitudes y emociones (KAASILA; HANNULA; LAINE; PEHKONEN, 2008; KRZYWACKI; HANNULA, 2010; CONTRERAS; PENALVA; TORREGROSA, 2011; LUTOVAC; KAASILA, 2011, 2013). Estos elementos dan lugar a la identidad profesional del maestro de matemáticas (mathematical identity work), que se interpreta como un proceso narrativo que incluye la interacción entre el contexto matemático individual y social y un proceso de auto-reflexión donde la identidad matemática pasada, presente y futura entran en diálogo (LUTOVAC; KAASILA, 2013).

Desde este marco, en este trabajo se considera que durante la formación inicial es fundamental conocer las creencias que conforman la identidad matemática profesional de los futuros maestros, y las creencias sobre la aptitud matemática de sus alumnos, puesto que 
dichas creencias pueden ejercer una notable influencia en el desempeño futuro de su profesión y, por consiguiente, en el aprendizaje de los alumnos (GIL; RICO, 2003; MINISTERIO DE EDUCACIÓN, CULTURA Y DEPORTE, 2012).

Contreras, Penalva y Torregrosa (2011) exponen que las identidades profesionales en formación de estudiantes para maestro emergen cuando reflexionan sobre su propia experiencia formativa en relación con la enseñanza y aprendizaje de las matemáticas. Así, pues, la identificación y toma de conciencia de estas creencias es el requisito necesario para poder promover procesos de cambio durante la formación inicial (KAASILA; HANNULA; LAINE, 2012), y evitar que los futuros maestros accedan a la práctica profesional con unas creencias negativas acerca de la aptitud de sus alumnos para aprender matemáticas, que acaben determinando el proceso de enseñanza-aprendizaje.

Es desde esta perspectiva que se ha desarrollado el presente estudio con un grupo de 142 futuros maestros de Infantil y Primaria, donde se analizan sus creencias sobre la aptitud matemática, es decir, los rasgos que para ellos distinguen un buen alumno de matemáticas de un mal alumno.

\section{Creencias sobre la aptitud para las matemáticas}

Para fundamentar teóricamente el análisis que se pretende llevar a cabo, se consideran los estudios sobre las agendas y ámbitos de investigación establecidos por Llinares (2008): Creencias y dominio afectivo: actitudes y cognición (Construcción del conocimiento y procesos matemáticos) y Aprender el conocimiento y destrezas útiles para enseñar matemáticas y desarrollo profesional. Variables y factores que influyen (El estudiante para profesor, el profesor y el formador de profesores: Aprendizaje y desarrollo profesional). Así pues, se considera tanto la literatura sobre el dominio afectivo y las creencias, como la que indaga acerca de los factores que influyen en el desarrollo profesional del profesorado.

En relación a las creencias, en este trabajo se asume la acepción de Gómez-Chacón, Op’t Eynde y De Corte (2006), según la cual una creencia nunca se sostiene con independencia de otras, por ello se suele hablar de sistemas de creencias y no de creencias aisladas. En el informe español del TEDS-M 2008 (MINISTERIO DE EDUCACIÓN, CULTURA Y DEPORTE, 2012) se señala que se trata de conocimientos subjetivos, convicciones generadas a nivel personal para explicar y justificar decisiones y actuaciones en el proceso de enseñanza-aprendizaje de las matemáticas. Estas creencias forman parte del dominio cognitivo, integran elementos afectivos, evaluativos y sociales, y presentan una 
fuerte estabilidad. Caballero, Blanco y Guerrero (2008) indican cuatro dimensiones del estudio de las creencias en educación matemática: creencias sobre la naturaleza de las matemáticas y de su enseñanza y aprendizaje, creencias sobre uno mismo como aprendiz de matemáticas, creencias acerca del papel del profesorado de matemáticas y creencias suscitadas por el contexto sociofamiliar.

Nuestro estudio se centra, principalmente, en el análisis de las creencias sobre uno mismo como aprendiz de matemáticas (atribución causal de éxito o fracaso en matemáticas) y las creencias acerca del papel del profesorado de matemáticas (la visión de las características personales de los alumnos y del papel del profesor en el proceso de enseñanza-aprendizaje).

Desde la psicología del aprendizaje, la influencia de las características personales (que son de naturaleza cognitiva y emocional, como por ejemplo la percepción de uno mismo, la motivación etc.) tradicionalmente ha sido explicada a partir de la existencia de diferencias individuales (MONEREO et al., 1994). Así, se establecía que un alumno es más inteligente que otro, como rasgo estable; que cada alumno posee unas características que facilitan o dificultan el aprendizaje y que existen alumnos más motivados que otros hacia las tareas escolares. Esta perspectiva es el producto de una tradición de investigación psicológica experimentalista, por una parte, y psicométrica, por otra.

La investigación en educación matemática, realizada mayoritariamente a partir de estudios descriptivos y enmarcados en un paradigma interpretativo, ha permitido identificar algunas creencias de los futuros maestros acerca de los rasgos que distinguen a un buen alumno de matemáticas de un mal alumno. Estos trabajos, relacionados sobre todo con el estudio de la actitud hacia las matemáticas y el autoconcepto matemático de los estudiantes, revelan principalmente la influencia de factores cognitivos y afectivos (CABALLERO; GUERRERO; BLANCO, 2007; MINISTERIO DE EDUCACIÓN, CULTURA Y DEPORTE, 2012; RUIZ DE GAUNA; GARCÍA; SARASUA, 2013) y, en menor medida, factores procedimentales (NCTM, 2000; GUIRADO et al., 2010).

Caballero, Guerrero y Blanco (2007) indican que, por lo general, los futuros maestros atribuyen el éxito en matemáticas a la actitud del profesorado hacia el alumno, a una mayor dedicación al estudio de dicha materia y al esfuerzo, descartando, en dicho éxito, la influencia de la suerte. Asocian tanto el éxito como el fracaso mayoritariamente a causas internas, inestables y controlables, y no consideran que la aptitud matemática reporte mayor valoración social por parte del grupo de iguales.

En el informe español del TEDS-M 2008 (MINISTERIO DE EDUCACIÓN, CULTURA Y DEPORTE, 2012) se señala que los futuros maestros tienden a considerar que 
el rendimiento en matemáticas depende, estrechamente, de la propia capacidad intelectual de cada alumno, es decir, algunos tienen capacidad natural para aprender matemáticas, mientras que otros no la tienen. El informe internacional denomina esta creencia Mathematics as a Fixed Ability, que significa que unos estudiantes son competentes en matemáticas y otros no, debido a capacidades intelectuales naturales e innatas.

Diversos estudios realizados con profesorado en ejercicio definen, con mayor precisión, las creencias sobre la aptitud matemática, es decir, los rasgos que para ellos distinguen un buen alumno de matemáticas de un mal alumno. Gil y Rico (2003), por ejemplo, indican que el criterio prioritario para determinar cuándo un alumno es bueno es su motivación, y señalan, también, otros rasgos específicos como tener buena capacidad intelectual, ser trabajador y ser responsable. Guirado et al. (2010) llegan a conclusiones similares con estudiantes de física, y destacan, sobre todo, las capacidades intelectuales (es inteligente, sabe estudiar, lee y entiende, razona, bueno en matemáticas); las actitudes y valores hacia la tarea (esfuerzo y trabajo, participativo, solidario, responsable); y el interés y las habilidades procedimentales (interés por la matemática, resuelve problemas), que en algunos casos mantienen cierto paralelismo con los procesos matemáticos descritos por el $\operatorname{NCTM}(2000)$.

Desde el punto de vista del desarrollo profesional del profesorado, Di Martino y Zan (2010) advierten de la necesidad de reflexionar con los futuros maestros acerca de su visión de las matemáticas y sus propias teorías sobre el éxito. A partir de las relaciones de los estudiantes con las matemáticas, elaboran un modelo que contempla la disposición emocional hacia las matemáticas (positiva o negativa), la visión de las matemáticas (relacional o instrumental) y la competencia matemática percibida (alta o baja). Los autores subrayan que los futuros maestros necesitan aprender cómo tratar estas tres dimensiones, por lo que es importante trabajar estos aspectos durante la formación de maestros como parte del conocimiento didáctico.

Kaasila, Hannula y Laine (2012) exponen que los estudios acerca de la visión de las matemáticas de los futuros maestros tienen un papel importante porque revelan cómo se va construyendo la identidad profesional del maestro de matemáticas, y, desde este marco, consideran que es necesario que los formadores de maestros comprendan sobre todo los puntos de vista negativos. Diversos estudios que han analizado las actitudes negativas hacia las matemáticas de los futuros maestros concluyen que se trata de un fenómeno generalizado (TRUJILLO; HADFIELD, 1999; HANNULA et al., 2005; OLIVEIRA; HANNULA, 2008), y señalan que estas emociones negativas podrían ser debidas a las experiencias previas como 
alumnos (TRUJILLO; HADFIELD, 1999; PIETILÄ, 2002; RUIZ DE GAUNA; GARCÍA; SARASUA, 2013).

Como ya se ha indicado, diversos estudios recientes (KRZYWACKI; HANNULA, 2010; CONTRERAS; PENALVA; TORREGROSA, 2011; LUTOVAC; KAASILA, 2011) señalan que estas experiencias y relaciones negativas con las matemáticas dan lugar a una identidad negativa, y Kaasila et al. $(2006,2008)$ apuntan que la toma de conciencia de esta identidad es el punto de partida necesario para promover procesos de cambio y de desarrollo en el profesorado.

En este contexto, el objetivo de nuestro estudio es identificar los factores que los futuros maestros de Educación Infantil y Primaria atribuyen a los buenos alumnos de matemáticas.

\section{Método}

Para determinar los factores que los estudiantes de Educación Infantil y Primaria consideran más importantes para ser un buen alumno de matemáticas, se analizan las creencias de 142 estudiantes para maestro, 72 pertenecientes al Grado de Educación Infantil y 70 del Grado de Educación Primaria. Todos los estudiantes, en el momento que se realizó el estudio, estaban cursando el primero en la Universidad de Girona (España).

La edad media de los estudiantes del Grado de Educación Infantil es de 21,2 años, y de los de Educación Primaria es de 20,3 años. El porcentaje de hombres en Educación Infantil es de 4,35\% y en Educación Primaria es de 12,04\%. El 56,5\% de los estudiantes de Infantil provienen del ciclo formativo de grado superior en Educación Infantil, y el 29,8\% de estudiantes de Primaria provienen de ciclos formativos de grado superior. De los estudiantes que provienen de bachillerato, el 14,28\% de Infantil y el 16,13\% de Primaria provienen de un bachillerato científico-tecnológico. Por lo tanto, de la muestra del estudio, solo el 4,4\% en Educación Infantil y el 6,8\% en Primaria, han cursado matemáticas en los dos últimos años antes de empezar los estudios universitarios.

A los individuos de la muestra se les pasó un cuestionario para identificar algunos datos básicos (edad, género, estudios anteriores) y diversas preguntas sobre su sistema de creencias acerca de las matemáticas, con una pregunta específica en la que se les pedía que indicaran tres factores (de mayor a menor importancia) que asociaban a un buen alumno de matemáticas. 
Dado el tipo de población y los datos analizados, aunque la pregunta a analizar sea una variable cualitativa, la metodología que se ha utilizado para su análisis y discusión ha sido de tipo cuantitativo. Para la categorización de las respuestas dadas, se ha combinado la categorización deductiva y la inductiva (BONILLA; RODRÍGUEZ, 2005), es decir, en un primer momento hemos partido de unidades de significado identificadas en marcos teóricos existentes (categorización deductiva) pero, posteriormente, con la revisión cuidadosa de todo el material, se han identificado subcategorías que emergen de la misma información (categorización inductiva).

Para el análisis de las respuestas dadas por los estudiantes para maestro, hemos clasificado los factores indicados en tres categorías principales. Estas categorías principales, se han subdividido en cuatro subcategorías.

Las tres categorías principales que se han considerado para clasificar las respuestas han sido: factores cognitivos, factores procedimentales y factores actitudinales, que se han establecido a partir de la revisión de la literatura. Entendemos los factores cognitivos como los que ayudan al estudiante a saber conocer. Esta categoría la hemos subdividido en cuatro subcategorías, definidas a partir de las respuestas dadas por los estudiantes y a partir de las categorías del trabajo de Guirado et al. (2010): inteligencia, memoria, comprensión y cálculo mental y escrito. Los factores procedimentales son aquellos que permiten al alumno saber hacer. En esta categoría, basándonos en los estándares de procesos definidos por el NCTM (2000), hemos considerado las subcategorías: razonamiento, comunicación, conexiones y resolución de problemas. Por último, entendemos como factores actitudinales los que ayudan al alumno a saber ser. Las cuatro subcategorías que hemos definido dentro de esta categoría, a partir de las categorías definidas por otros autores como Guirado et al. (2010) y las respuestas dadas por los estudiantes, han sido: interés, esfuerzo, participación y autoconcepto.

Para tener en cuenta el orden de los tres factores que indican los estudiantes como más importantes para ser un buen alumno en matemáticas (de mayor a menor), se han ponderado sus respuestas dando un peso de 0,5 al primer factor, de 0,3 al segundo y de 0,2 al factor que indican en tercera posición.

\section{Resultados}

Para la exposición de los resultados, en primer lugar se muestran los resultados generales por categorías, comparando las respuestas de los estudiantes de Educación Infantil con las de los estudiantes de Educación Primaria. En segundo lugar, se muestran los 
resultados obtenidos en las subcategorías de cada categoría, definidas y explicadas anteriormente. De la misma manera, en este segundo bloque de resultados, también se han comparado los estudiantes de Educación Infantil con los de Educación Primaria.

Tal como se ha justificado, al ponderar las respuestas de los estudiantes según el orden de los factores indicados $(0,5 ; 0,3 ; 0,2)$, el número de estudiantes que se indican en las tablas de resultados de cada categoría o subcategoría, no es un número natural.

\subsection{Resultados por categorías}

En primer lugar, mostramos los resultados que hemos obtenido de las respuestas de los alumnos de Educación Infantil y Primaria sobre los tres factores que consideran de mayor relevancia para ser un buen alumno en matemáticas. Hemos clasificado sus respuestas según si son factores cognitivos, factores procedimentales o factores actitudinales.

Tabla 1- Resultados obtenidos en las tres categorías

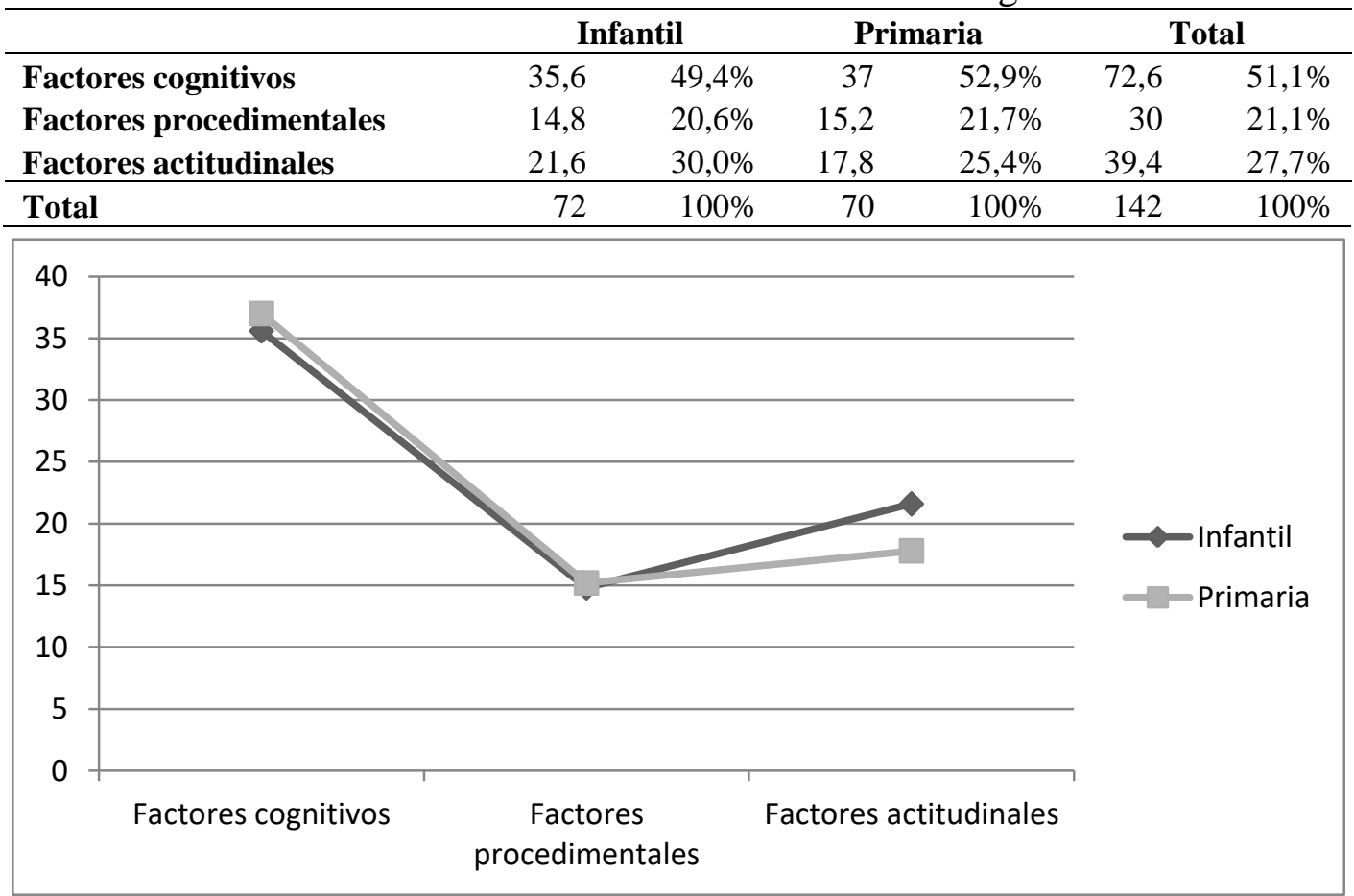

Figura 1- Gráfico comparativo entre los estudiantes de Educación Infantil y Primaria en las tres categorías

Estos resultados muestran que casi la mitad de los estudiantes (en Infantil un $49 \%$ y en Primaria un 53\%), le da mayor importancia a los factores cognitivos cuando se les pregunta por los rasgos que definen a un buen alumno en matemáticas. En segundo lugar, siendo de un $30 \%$ entre los estudiantes de Infantil y de un $25 \%$ en Primaria, consideran los factores actitudinales. Los factores que menos atribuyen como necesario en un buen alumno de matemáticas, en los dos casos son los procedimentales. 


\subsection{Resultados por subcategorías}

A continuación se presentan los resultados de forma más detallada, diferenciando, dentro de cada categoría anterior, cuatro sub-categorías. De la misma forma que en los resultados anteriores, se presentan los resultados de los estudiantes de Infantil y de los de Primaria por separado, comparando así estos dos grupos de población.

Tabla 2 - Resultados obtenidos en las 12 subcategorías

\begin{tabular}{clrrrrrr}
\hline & & \multicolumn{2}{c}{ Infantil } & \multicolumn{2}{c}{ Primaria } & \multicolumn{2}{c}{ Total } \\
\hline & Inteligencia & 9,2 & $12,8 \%$ & 17,6 & $25,1 \%$ & 26,8 & $18,9 \%$ \\
Factores & Memoria & 1,8 & $2,5 \%$ & 2 & $2,9 \%$ & 3,8 & $2,7 \%$ \\
cognitivos & Comprensión & 4,6 & $6,4 \%$ & 7,8 & $11,1 \%$ & 12,4 & $8,7 \%$ \\
& Cálculo mental y escrito & 20,3 & $28,2 \%$ & 10,9 & $15,6 \%$ & 31,2 & $22,0 \%$ \\
& Razonamiento & 3,1 & $4,3 \%$ & 2,1 & $3,0 \%$ & 5,2 & $3,7 \%$ \\
Factores & Comunicación & 0,7 & $1,0 \%$ & 1,5 & $2,1 \%$ & 2,2 & $1,5 \%$ \\
procedimentales & Conexiones & 3,2 & $4,4 \%$ & 2,9 & $4,1 \%$ & 6,1 & $4,3 \%$ \\
& Resolución de problemas & 8,4 & $11,7 \%$ & 8,7 & $12,4 \%$ & 17,1 & $12,0 \%$ \\
& Interés & 10,7 & $14,9 \%$ & 8,8 & $12,6 \%$ & 19,5 & $13,7 \%$ \\
Factores & Esfuerzo & 8,2 & $11,4 \%$ & 6,4 & $9,1 \%$ & 14,6 & $10,3 \%$ \\
actitudinales & Participación & 0,5 & $0,7 \%$ & 0,2 & $0,3 \%$ & 0,7 & $0,5 \%$ \\
& Autoconcepto & 1,3 & $1,8 \%$ & 1,1 & $1,6 \%$ & 2,4 & $1,7 \%$ \\
\hline & Total & 72 & $100 \%$ & 70 & $100 \%$ & 142 & $100 \%$ \\
\hline
\end{tabular}

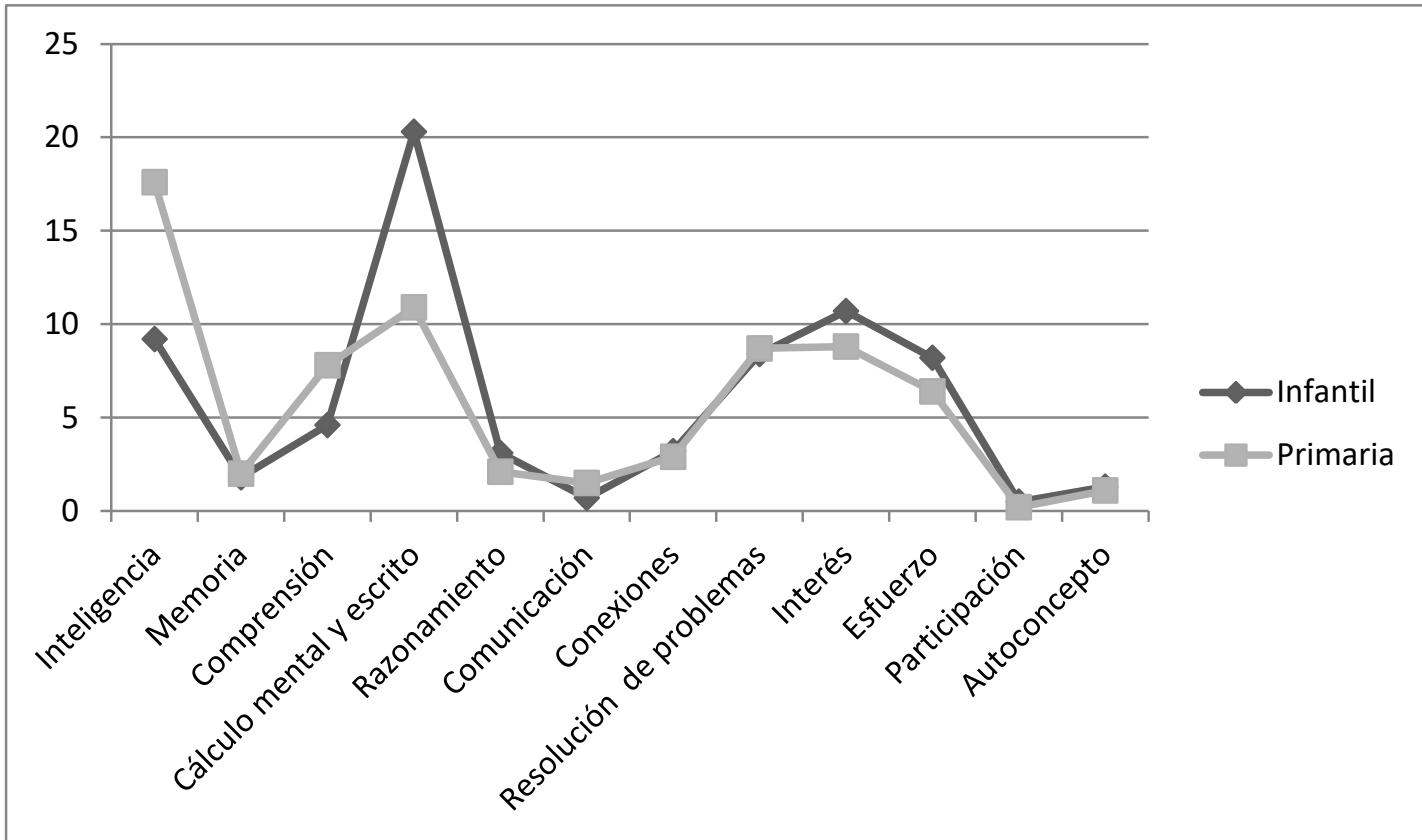

Figura 2 - Gráfico comparativo entre los estudiantes de Educación Infantil y Primaria en las subcategorías

En la tabla 2 y el gráfico 2, respectivamente, se observa que los estudiantes de Educación Infantil (un 28\%) consideran que el factor más importante para ser un buen alumno de matemáticas es tener un buen dominio del cálculo mental y escrito (factor cognitivo). En cambio, los estudiantes de Educación Primaria (25\%) consideran más importante ser inteligente (también es un factor cognitivo). En segundo lugar, los estudiantes de Infantil (un 
15\%), han destacado un factor actitudinal, tener interés en cambio; en cambio, los estudiantes de Primaria (casi un 16\%), en segundo lugar también destacan otro factor cognitivo, ser bueno en el cálculo mental y escrito.

Dentro de la categoría de factores procedimentales, el factor que consideran más importante en los dos grupos de población es la resolución de problemas (en los dos casos un 12\%). En la categoría de factores actitudinales, tal como se ha indicado, los estudiantes de Educación Infantil le han dado más importancia a que los alumnos tengan interés por lo que estudian (un 15\%) igual que los estudiantes de Educación Primaria, aunque en menor medida (no llega al 13\%).

Para los estudiantes de Educación Infantil y de Educación Primaria, los factores que consideran menos importantes (o los que menos estudiantes han indicado como importantes) para ser un buen alumno en matemáticas, son la participación, comunicación y Autoconcepto (menos de un $2 \%$ ).

\section{Discusión y conclusiones}

En la literatura sobre el dominio afectivo en educación matemática existe cierto consenso acerca de la influencia que ejercen las creencias en la práctica docente del maestro y, por consiguiente, en el aprendizaje de los alumnos (GROUWS, 1996; CARTER; NOREOOD, 1997; BROWN, 2003; GIL; RICO, 2003; KAYAN, 2007; MINISTERIO DE EDUCACIÓN, CULTURA Y DEPORTE, 2012).

Estas creencias, como se ha indicado, forman parte de la identidad matemática que se va construyendo a la largo de la vida a partir de las relaciones que se establecen con las matemáticas (KAASILA et al., 2008; KRZYWACKI; HANNULA, 2010; CONTRERAS; PENALVA; TORREGROSA, 2011; LUTOVAC; KAASILA, 2011, 2013), y originan la identidad profesional del maestro de matemáticas (LUTOVAC; KAASILA 2013), que acaba determinando lo que el maestro hace en el aula.

Desde este prisma, un primer aspecto novedoso de nuestro estudio respecto a investigaciones previas es que ha permitido identificar globalmente todos los factores cognitivos, actitudinales y procedimentales - que los futuros maestros atribuyen a los buenos alumnos de matemáticas, para poder transformarlos durante la formación inicial (KAASILA; HANNULA; LAINE, 2012), sobre todo si dan lugar a una identidad negativa (KAASILA et al., 2006, 2008). 
Otro dato revelador que aporta nuestro estudio es la definición de las subcategorías incluidas en cada una de las tres categorías de factores establecidas, y más concretamente, el peso específico de cada subcategoría. En el conjunto de subcategorías de los factores cognitivos, los estudiantes del Grado de Educación Infantil piensan que el factor que identifica mejor a un buen alumno de matemáticas es el cálculo mental y escrito, y en segundo lugar la inteligencia, que es el primer factor que manifiestan los estudiantes de Primaria. Si bien la inteligencia es un factor recurrente en la literatura (NCTM, 2000; GIL; RICO, 2003; GUIRADO et al., 2010; MINISTERIO DE EDUCACIÓN, CULTURA Y DEPORTE, 2012), nos sorprende el dato respecto a los estudiantes de Infantil en relación a la importancia que otorgan al cálculo mental y escrito. Una posible interpretación de este dato podría atribuirse al hecho de que consideran que las matemáticas son, sobre todo, hacer cálculos. En ambos grupos de estudiantes, la memoria parece ser la variable que tiene menor peso en el conjunto de los factores cognitivos.

En relación a los factores actitudinales, las subcategorías que parecen ser más predictivas son el interés por la materia y el esfuerzo, mientras que el autoconcepto tiene una escasa valoración. Estos datos coinciden con los resultados del estudio de Caballero, Guerrero y Blanco (2007), que subrayan la importancia que los futuros maestros dan al estudio de la materia y al esfuerzo, a la vez que no consideran que la aptitud matemática reporte mayor valoración social.

En relación a los factores procedimentales, los futuros maestros consideran que la resolución de problemas es el rasgo que define mejor a un buen alumno de matemáticas, mientras que la comunicación es el proceso de pensamiento matemático al que otorgan menor importancia. Respecto a estos factores, sorprende el escaso peso que le otorgan los futuros maestros en el conjunto de creencias acerca de la aptitud matemática, frente a los factores cognitivos y actitudinales.

Aunque la pretensión inicial del estudio no era ésta, los datos evidencian que, para los futuros maestros, no parece demasiado importante que un buen alumno de matemáticas tenga buenas habilidades en relación a las formas de adquisición y uso de los contenidos matemáticos. Estos datos se contradicen con las tendencias contemporáneas en educación matemática (NCTM, 2000) que ponen de relieve la importancia de los procesos de pensamiento matemático para el aprendizaje de las matemáticas, en general, y para la adquisición de la competencia matemática, en particular.

En conclusión, nuestros resultados señalan que los futuros maestros atribuyen tres tipos de factores a los buenos alumnos de matemáticas: en primer lugar consideran los 
factores cognitivos (inteligencia-memoria), en segundo lugar los factores actitudinales (interés-autoconcepto) y en tercer lugar los factores procedimentales (resolución de problemas-comunicación). La incorporación de los factores procedimentales implica realizar nuevos estudios que contribuyan a definir, con más precisión, los factores de cada categoría, y que permitan concretar los que tienen mayor peso en el conjunto de creencias acerca de la aptitud matemática. A partir de su diagnóstico será posible trabajar con precisión durante la formación inicial, como parte del conocimiento didáctico, las creencias que dan lugar a una identidad profesional negativa. Todo ello para formar a los futuros maestros de matemáticas de acuerdo con el principio de igualdad en la educación matemática, que requiere altas expectativas y un fuerte apoyo para todos los estudiantes.

\section{Referencias}

BONILLA, E.; RODRÍGUEZ, P. Más allá del dilema de los métodos. Colombia: Editorial Nomos, 2005.

BROWN, N. M. . A study of elementary teachers' abilities, attitudes, and beliefs about problem solving.2003. Dissertation Abstracts Disponible en:

<http://digitalcommons.georgiasouthern.edu/etd_legacy/691>. Accesso em: 13 oct. 2016

CABALLERO, A.; BLANCO, L. J.; GUERRERO, E. El dominio afectivo en futuros maestros de matemáticas en la Universidad de Extremadura. Paradigma, Maracay, n. 24(2), p. 157-171, 2008.

CABALLERO, A.; GUERRERO, E.; BLANCO, L. J. Las actitudes y emociones ante las Matemáticas de los estudiantes para Maestros de la Facultad de Educación de la Universidad de Extremadura. In: CAMACHO, M. et al.(Ed.). Investigación en Educación Matemática. Tenerife: SEIEM, 2007. p 41-42.

CARRILLO, J. Modos de resolver problemas y concepciones sobre la matemática y su enseñanza de profesores de matemáticas de alumnos de más de 14 años: algunas aportaciones a la metodología de la investigación y estudio de posibles relaciones. Revista de la sociedad Andaluza de Educación matemática "Thales", Huelva, n. 36, p. 451-454, 1998.

CARTER, G.; NORWOOD, K.S. The relationship between teacher and student's beliefs about mathematics. School Science and Mathematics, Malden, n. 97(2), p. 62-67, 1997

CONTRERAS, L. C. Concepciones de los profesores sobre la resolución de problemas. Huelva: Universidad de Huelva, 1999.

CONTRERAS, P.; PENALVA, M. C.; TORREGROSA, G. (2011). Identidad profesional y conocimiento matemático para la enseñanza de maestros en formación. In: MARÍN, M. et al. (Ed.). Investigación en Educación Matemática. Ciudad Real: SEIEM, 2011.p. 329-338.

DI MARTINO, P.; ZAN, R. "Me and maths": towards a definition of attitude grounded on students' narratives. Journal of Mathematics Teacher Education, Dordrech, n. 13(1), p. 27-48, 2010.

GIL, F.; RICO, L. Concepciones y creencias del profesorado de secundaria sobre enseñanza y aprendizaje de las matemáticas. Enseñanza de las Ciencias, Valencia, n. 21(1), p. 27-47, 2003. 
GÓMEZ-CHACÓN, I. M.; OP’T EYNDE, P.; DE CORTE, E. Creencias de los estudiantes de matemáticas. La influencia del contexto de clase. Enseñanza de las Ciencias, Valencia, n. 24(3), p. 309- 324, 2006.

GROUWS, D. A. Critical issues in problem solving instruction in mathematics. In: ZHANG, D.; SAWADA, T.; BECKER, J. P. (Ed.). Proceedings of the China-Japan-U. S. seminar on mathematical education . Carbondale, IL: Southern Illinois University, 1996, p.70-93.

GUIRADO, A. M.; OLIVERA, A. C.; MAZZITELli, C. A.; AGUILAR, S. B. ¿Cuál es la representación que tienen los docentes acerca de ser un buen alumno de física y aprender física? Revista Electrónica de Enseñanza de las Ciencias, Valencia, n. 9(3), p. 618-632, 2010.

HANNULA, M. S.; KAASILA, R.; LAINE, A.; PEHKONEN, E. Structure and typical profiles of elementary teacher students' view of mathematics. In: CHICK, H. L.; J. L. Vincent (Ed.). PROCEEDINGS OF THE CONFERENCE OF THE INTERNATIONAL GROUP FOR THE PSYCHOLOGY OF THE EDUCATION, 29., 2005, Melbourne. Anales... Melborune: PME, 2005. p. 89-96.

KAASILA, R.; HANNULA, M.; LAINE, A.; PEHKONEN, E. Facilitators for change of elementary teacher students' view of mathematics. In: NOVOTANÁ, J.; MORAOVÁ, H.;KRÁTKÁ, M.; STEHLIKOVÁ, N. (Ed.). PROCEEDINGS OF THE CONFERENCE OF THE PSYCHOLOGY OF THE MATHEMATICS EDUCATION, 30., 2006, Praga. Anales... Praga: PME, 2006. p. 385-392.

KAASILA, R.; HANNULA, M.; LAINE, A.; PEHKONEN, E. Socio-emotional orientations and teacher change. Educational Studies in Mathematics, New York, n. 67, p. 111-123, 2008.

KAASILA, R.; HANNULA, M.; LAINE, A. "My personal relationship towards mathematics has necessarily not changed but..." Analyzing pre-service teachers' mathematical identity talk.

International Journal of Science and Mathematics Education, Taiwan, n. 10, p. 975-995, 2012.

KAYAN, F. A study on preservice elementary mathematics teachers' mathematical problem solving beliefs. 2007. Tesis Doctoral-Üniversitesi Fen Bilimleri Enstitüsü, Ankara, 2007].

KRZYWACKI, H.; HANNULA, M. S. Tension between present and ideal state of teacher identity in the core of professional development. In: PINTO, M. M. F.; KAWASAKI, T. F. (Ed.), PROCEEDINGS OF THE CONFERENCE OF THE PSYCHOLOGY OF THE MATHEMATICS EDUCATION, 34, 2010, Belo Horizonte. Anales... Belo Horizonte: PME, 2010. p. 267-271.

LLINARES, S. Agendas de investigación en educación matemática en España: una aproximación desde "ISI-web of knowledge" y ERIH". In: LUENGO, R.; GÓMEZ, B.; CAMACHO, M.; BLANCO, L. (Ed.). Investigación en Educación Matemática. Badajoz: SEIEM, 2008. p. 25-54..

LUTOVAC, S.; KAASILA, R. Beginning a pre-service teacher's mathematical identity work through narrative rehabilitation and bibliotherapy. Teaching in Higher Education, London, n. 16(2), p. 225236, 2011.

LUTOVAC, S.; KAASILA, R. Pre-service teacher's possible mathematical identities. Acceso en: $<$ http://blogs.helsinki.fi/mavi-2012/files/2012/09/Lutovac Kaasila_MAVI-2012_revised-for-theweb2.doc $>$.

MINISTERIO DE EDUCACIÓN, CULTURA Y DEPORTE. TEDS-M. Informe español. Estudio internacional sobre la formación inicial en matemáticas de los maestros. Madrid: Secretaría General Técnica. Subdirección General de Documentación y Publicaciones. 2012. 
MONEREO, C. et al. Análisis de los factores que intervienen en la enseñanza-aprendizaje de estrategias en el aula. In: MONEREO, C. (Ed.). Estrategias de Enseñanza y Aprendizaje. Barcelona: Graó, 1994. p. 75-98.

NCTM-NATIONAL COUNCIL OF TEACHERS OF MATHEMATICS. Principles and standards for school mathematics. Reston, VA: The National Council of Teachers of Mathematics. 2000.

OLIVEIRA, H.; HANNULA, M. S. Individual prospective mathematics teachers. In: KRAINER, K.; WOOD, T. (Ed.).Participants in mathematics teacher education: Individuals, teams, communities and networks. Rotterdam: Sense, 2008. p.13-34.

PIETILÄ, A. The role of mathematics experiences in forming pre-service elementary teachers' views of mathematics. In: COCKBURN, A.; NARDI, E. (Ed.), CONFERENCE OF THE INTERNATIONAL GROUP FOR THE PSYCHOLOGY OF MATHEMATICS EDUCATION, 26., 2002, Norwich. Proceedings... Norwich: PME, 2002. p. 57-64.

PONTE, J. P. Las creencias y concepciones de maestros como un tema fundamental en formación de maestros. In:KRAINER, K.; GOFFREE, F. (Ed.). On research in teacher education: from a study of teaching practices to issues in teacher education. Osnabrïck: Forschungsintitut für Mathematikdidaktik, 1999. p. 43-50.

RUIZ DE GAUNA, J.; GARCÍA, J.; SARASUA, J. Perspectiva de los alumnos de Grado de Educación Primaria sobre las Matemáticas y su enseñanza. Números, La Laguna, n. 82, p. 5-15, 2013.

THOMSON, A. G. Teachers' beliefs and conceptions: A synthesis of the research. In: GROUWS, D. A. (Ed.). Handbook of research in mathematics learning and teaching. Nueva York: McMillan, 1992. p. 127-146.

TRUJILLO, K. M.; HADFIELD, O. D.Tracing the roots of mathematics anxiety through in-depth interviews with preservice elementary teachers. College Student Journal, Mobile, Alabama], v. 11, n. 33, 219-232, 1999.

Submetido em Abril de 2015. Aprovado em Março de 2016. 